\title{
Optimal Detection of Subtle Gadolinium Leakage in CSF with Heavily T2-Weighted Fluid-Attenuated Inversion Recovery Imaging
}

(D)W.M. Freeze, (DD.N. ter Weele, (D)W.M. Palm, DR.W. van Hooren, DE.I. Hoff, (D).F.A. Jansen, (DH.I.L. Jacobs, DF.R. Verhey, and

(D)W.H. Backes

\begin{abstract}
SUMMARY: Pericortical enhancement on postcontrast FLAIR images is a marker for subtle leptomeningeal blood-brain barrier leakage. We explored the optimal FLAIR sequence parameters for the detection of low gadolinium concentrations within the CSF. On the basis of phantom experiments and human in vivo data, we showed that detection of subtle pericortical enhancement can be facilitated by using a relatively long TE. Future studies should choose their FLAIR sequence parameters carefully when assessing pericortical enhancement due to subtle blood-brain barrier leakage.
\end{abstract}

ABBREVIATIONS: $B B B=$ blood-brain barrier; $\mathrm{pc}=$ postcontrast

P ostcontrast (pc) FLAIR imaging can be applied to visualize leakage of contrast material through the blood-brain barrier (BBB) into the CSF. ${ }^{1}$ This technique is becoming increasingly popular because of its superior sensitivity for detecting low gadolinium concentrations in CSF (but not cerebral tissue) compared with pcT1WI. ${ }^{2}$ Another advantage of pcFLAIR imaging over pcT1WI is the suppression of signal from blood containing high gadolinium concentrations, which makes it easier to discern leakage from leptomeningeal vessels. Previous studies have shown contrast agent extravasation in the form of CSF enhancement within the ventricular and/or pericortical space in various neurologic and cardiovascular surgery conditions $s^{3-8}$ and in cognitively healthy older individuals. ${ }^{2}$ Although the precise clinical relevance

Received May 10, 2019; accepted after revision June 19

From the Department of Psychiatry and Neuropsychology (W.M.F., R.W.v.H., H.I.L.J., F.R.V.), Maastricht University, School for Mental Health and Neuroscience, Alzheimer Centrum Limburg, Maastricht, the Netherlands; Departments of Radiology (D.N.t.W.) and Neurology (E.I.H.), Zuyderland Medical Center Heerlen, Heerlen, the Netherlands; and Department of Radiology and Nuclear Medicine (W.M.F., W.M.P., J.F.A,., W.H.B.), Maastricht University Medical Center, School for Mental Health and Neuroscience, Maastricht, the Netherlands.

Whitney M. Freeze and David N. ter Weele contributed equally to this work. This research was supported by Alzheimer Nederland and Stichting 2Bike4Alzheimer (research grant WE-03-2012-40).

Paper previously presented, in part, at: Annual Meeting of NVKF Lustrumcongres, April 4-5, 2019; Woudschoten, the Netherlands.

Please address correspondence to Walter H. Backes, PhD, Department of Radiology \& Nuclear Medicine, School for Mental Health and Neuroscience, Maastricht University Medical Center, PO Box 5800, 6202AZ Maastricht, the Netherlands; e-mail:w.backes@mumc.nl

$\equiv$ Indicates article with supplemental on-line appendix and table.

Indicates article with supplemental on-line photos.

http://dx.doi.org/10.3174/ajnr.A6145 and pathophysiology of pcFLAIR enhancement remains unclear, it has been associated with several adverse conditions, including hemorrhagic transformation after ischemic stroke ${ }^{5}$ and inflammation and reduced cortical thickness in MS. ${ }^{3,4}$

The conspicuity of pericortical enhancement depends on the specific contrast parameters of the pulse sequence and the time delay from contrast agent administration to pcFLAIR acquisition. Because BBB leakage is very subtle in many disease conditions, low concentrations of leaked contrast agent in the CSF may be missed by the neuroradiologist if the acquisition parameters or postcontrast acquisition timing or both are not tailored to account for BBB leakage. We explored the optimal FLAIR sequence parameters at 3T MR imaging to visualize low gadolinium concentrations within the CSF space using phantom experiments. Subsequently, we compared a regular pcFLAIR sequence with an optimized heavily T2-weighted pcFLAIR sequence. Both FLAIR scans were $3 \mathrm{D}$ sequences and were acquired at 2 time points, within a short timeframe after contrast injection and a $>1.5$-hour delay.

\section{MATERIALS AND METHODS \\ In Vitro Experiment}

We used phantoms with various aqueous gadolinium concentrations mimicking a range of leakage magnitude in CSF and tissue. See our previous publication ${ }^{2}$ and the On-line Appendix for more details.

\section{Participants and MR Imaging Protocol}

We prospectively included 6 older individuals who presented with pericortical CSF enhancement in our baseline study, and images were obtained on the same scanner. ${ }^{2}$ There was no overlap be- 


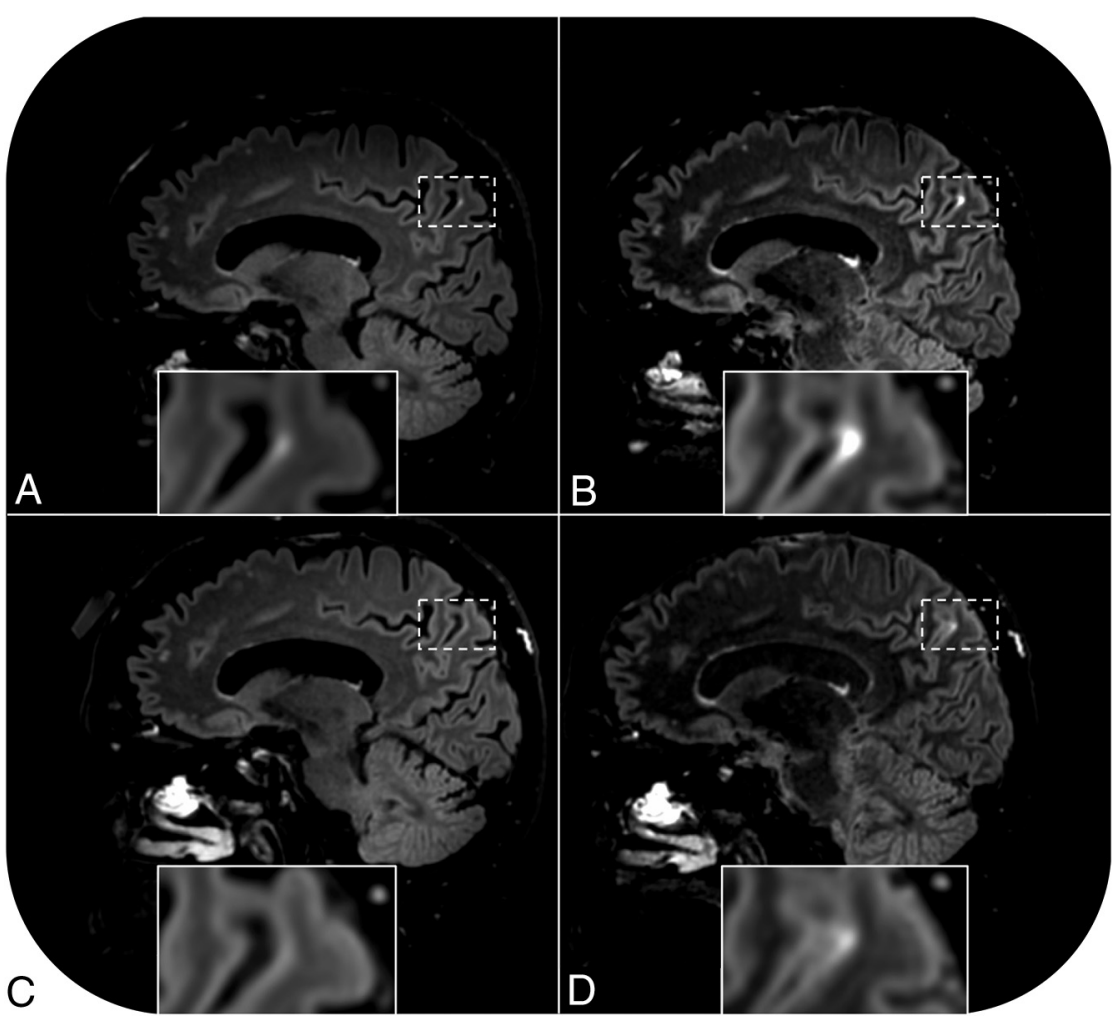

FIGURE. Pericortical enhancement is visible on postcontrast FLAIR images of a 68-year-old cognitively healthy female participant (case 1). Enhancement is apparent on the regular postcontrast FLAIR scan acquired 16 minutes after contrast administration $(A)$ and even more conspicuous on the heavily T2-weighted FLAIR scan acquired immediately thereafter $(B)$. The sequences were repeated 131 minutes after contrast administration. No signal enhancement is visible on the repeat regular postcontrast FLAIR scan $(C)$, but enhancement is still visible on the repeat heavily T2weighted FLAIR scan (D).

tween the follow-up participants in the baseline study $(n=3)$ and the present work $(n=6)$. During the first scan session, a regular precontrast FLAIR scan was acquired. Subsequently, 16 minutes after intravenous administration of $0.1 \mathrm{mmol} / \mathrm{kg}$ of gadobutrol, the regular FLAIR sequence $(\mathrm{TR} / \mathrm{TE} / \mathrm{TI}=4800 / 290 / 1650 \mathrm{~ms})$ was repeated, followed by a heavily T2-weighted FLAIR sequence $(\mathrm{TR} / \mathrm{TE} / \mathrm{TI}=4800 / 500 / 1650 \mathrm{~ms})$. Both pcFLAIR sequences were repeated in the same order during a second (later) scan session after at least a 1.5-hour delay (range, 100-263 minutes). Additional information on FLAIR sequence details and other MR imaging sequences used to evaluate cerebrovascular damage are described in the On-line Appendix. Informed consent was obtained from all participants before participation. This study was performed in accordance with the local institutional review boards (Zuyderland Medical Center Heerlen and Maastricht University Medical Center) and approved by the medical ethics committee in Maastricht.

\section{Image Analysis}

Pericortical enhancement was independently evaluated by 2 raters (the same as in our baseline study) ${ }^{2}$ blinded to clinical data, acquisition time, and scan parameters on all FLAIR scans (including the precontrast FLAIR scans as a negative control). The number of hyperintense foci was counted on each scan. Discrepancies were evaluated by consensus agreement. All images were checked for changes in MR imaging markers of cerebrovascular pathology compared with baseline by the same experienced neuroradiologist and methods as previously described. ${ }^{2}$

\section{RESULTS}

\section{In Vitro Experiment}

The results of our phantom experiments show 2 important concepts that facilitate the detection of low gadolinium concentrations within the CSF, namely the use of relatively long TEs and proper nulling of pure CSF. These concepts as well as the influence of each sequence parameter on the image appearance are further explained in the On-line Appendix and in On-line Figs 1-4.

\section{Neuroradiologic Imaging}

The median age of our participants was 73 years (range, 68-84 years), and 4 of 6 were men. All baseline foci of signal enhancement had remained stable with time, and one de novo leakage focus was detected on the regular pcFLAIR images acquired during the first scan session (the total number of pericortical hyperintensities was 9, and in addition, enhancement was observed within an enlarged perivascular space; On-line Table). Pericortical enhancement appeared visually more intense on all heavily T2-weighted pcFLAIR images compared with the regular pcFLAIR images. In 4 of 6 cases, the enhancing foci $(n=6)$ were visible on all pcFLAIR scans. In 1 case (case 1), a pericortical hyperintensity was visible on all pcFLAIR scans except on the regular pcFLAIR scan that was acquired during the later scan session (Figure). In another case (case 2), two additional foci of enhancement were visible on the heavily T2weighted FLAIR scan of the first scan session that were not visible on the regular pcFLAIR scan, but all foci had disappeared on both types of pcFLAIR scans that were acquired during the later session (On-line Table). In contrast, widespread signal enhancement within the sulci of another participant (case 3) only became clearly visible on the heavily T2-weighted (but not the regular) pcFLAIR scan in the later scan session (On-line Fig 5). This enhancement resembled the acute lesion-related hyperacute injury marker that has been reported in earlier studies. ${ }^{5,6}$ Most interesting, this case showed a new cerebral microbleed within the thalamus and a region of recent ischemia without cavitation within the basal ganglia that were not present at baseline. No cerebrovascular changes were detected in the other participants compared with baseline, and no enhancement was detected on the precontrast FLAIR images.

\section{DISCUSSION}

In the present study, we explored the optimal 3T MR imaging FLAIR sequence parameters to visualize low gadolinium concen- 
trations within the CSF space and compared a regular pcFLAIR sequence with a more optimal heavily T2-weighted pcFLAIR sequence acquired at 2 time points after contrast administration. Our results show increased conspicuity of pericortical hyperintensities due to subtle BBB leakage on the heavily T2-weighted pcFLAIR sequence because the strong T2-weighting suppresses the tissue signal, which emphasizes the appearance of CSF enhancement due to contrast agent leakage. In addition, we emphasized the importance of proper nulling of pure CSF, which also facilitates the detection of subtle gadolinium leakage.

Previous studies that assessed BBB leakage of gadoliniumbased contrast agents into the CSF with 3T MR imaging used diverse acquisition parameters; these make it difficult to compare the results across various studies, such as the prevalence and number of foci of pericortical enhancement within a specific neurologic disease. Furthermore, previous studies acquired their postcontrast FLAIR scans at varying time points after contrast administration, another factor that influences the appearance of pericortical enhancement. Our results provide guideline acquisition parameters for future studies that are interested in measuring subtle gadolinium leakage in CSF. With regard to acquisition timing, our results show that focal enhancement visible within a short timeframe after contrast administration can disappear at a later time, probably because blood gadolinium concentrations and thereby leakage concentrations decrease with time. However, we also showed 1 patient with neuroradiologic evidence of recent ischemic and hemorrhagic brain lesions who had diffuse gadolinium leakage that only became apparent after a 2-hour delay. Thus, widespread signal enhancement within the sulci in the context of recent or semirecent cerebrovascular damage may become more clearly visible at a later acquisition stage due to delayed and/or cumulative gadolinium leakage, possibly through cerebrovascular clearance pathways.

An obvious limitation of this study is the small sample size and variability in the time delay between the first and second imaging session, which hampers drawing firm conclusions on differences in the number of pericortical leaks that can be detected with either FLAIR sequence. Future studies should explore differences in the number of pericortical leaks that can be detected with either FLAIR sequence further, along with the dynamic mechanisms underlying focal (probably chronic) and widespread (ie, hyperacute injury marker-like, possibly postacute lesion-related) signal enhancement on pcFLAIR images. Most important, pcFLAIR CSF signal enhancement forms a promising biomarker for subtle BBB leakage that can be visually assessed by a neuroradiologist and could be used to monitor and evaluate treatment efficacy in future clinical trials that target cerebrovascular integrity.

\section{CONCLUSIONS}

The detection of subtle BBB leakage of gadobutrol into the CSF can be improved by using a heavily T2-weighted pcFLAIR sequence. Future studies that aim to detect low gadolinium concentrations within the CSF should consider the dynamic aspect of the enhancement and choose their FLAIR sequence parameters and acquisition timing carefully.

Disclosures: Whitney M. Freeze-RELATED: Grant: Alzheimer Nederland and Stichting 2Bike4Alzheimer, Comments: Costs related to data acquisition and part of my (regular) salary were covered by Alzheimer Nederland and Stichting 2Bike4Alzheimer (research grant WE-03-2012-40).* Roy W. van Hooren—RELATED: Alzheimer Nederland (research grant WE-03-2012-40), Comments: Part of my salary enabling my work for this project was funded by this grant*; UNRELATED: Employment: Maastricht University, Comments: The rest of my salary was paid by Maastricht University. Heidi I.L. Jacobs-RELATED: Grant: Alzheimer Nederland, Comments: This research was supported by Alzheimer Nederland (research grant WE-03-2012-40)*; Support for Travel to Meetings for the Study or Other Purposes: Alzheimer Nederland, Comments: Dr Freeze was supported by Alzheimer Nederland to present this work July 14-18, 2019 at Alzheimer's Association International Conference. * Frans R. VerheyUNRELATED: Grants/Grants Pending: European Commission, Comments: Dementia: Intersectorial Strategy for Training and Innovation Network for Current Technology, Marie Curie H2020 Innovative Training Network. * Money paid to institution.

\section{REFERENCES}

1. Köhrmann M, Struffert T, Frenzel T, et al. The hyperintense acute reperfusion marker on fluid-attenuated inversion recovery magnetic resonance imaging is caused by gadolinium in the cerebrospinal fluid. Stroke 2012;43:259-61 CrossRef Medline

2. Freeze WM, Schnerr RS, Palm WM, et al. Pericortical enhancement on delayed postgadolinium fluid-attenuated inversion recovery images in normal aging, mild cognitive impairment, and Alzheimer disease. AJNR Am J Neuroradiol 2017;38:1742-47 CrossRef Medline

3. Absinta M, Vuolo L, Rao A, et al. Gadolinium-based MRI characterization of leptomeningeal inflammation in multiple sclerosis. $\mathrm{Neu}$ rology 2015;85:18-28 CrossRef Medline

4. Zivadinov R, Ramasamy DP, Vaneckova M, et al. Leptomeningeal contrast enhancement is associated with progression of cortical atrophy in MS: a retrospective, pilot, observational longitudinal study. Mult Scler 2017;23:1336-45 CrossRef Medline

5. Latour LL, Kang DW, Ezzeddine MA, et al. Early blood-brain barrier disruption in human focal brain ischemia. Ann Neurol 2004;56: 468-77 CrossRef Medline

6. Kidwell CS, Burgess R, Menon R, et al. Hyperacute injury marker (HARM) in primary hemorrhage: a distinct form of CNS barrier disruption. Neurology 2011;77:1725-28 CrossRef Medline

7. Merino JG, Latour LL, Tso A, et al. Blood-brain barrier disruption after cardiac surgery. AJNR Am J Neuroradiol 2013;34:518-23 CrossRef Medline

8. Eisele P, Griebe M, Szabo K, et al. Investigation of leptomeningeal enhancement in MS: a postcontrast FLAIR MRI study. Neurology 2015;84:770-75 CrossRef Medline 